\title{
Simulación clínica en pediatría y neonatología: adaptación telemática del proyecto de innovación educativa.
}

Evelin Balaguer López ${ }^{\text {ab }}$, Pablo Buck Sainz-Rozasa, Manuel Ruescas López ${ }^{a b}$, Carmen Casal Angulo $^{\text {ac }}$, Pedro García Martínez ${ }^{\mathrm{d}}$ y Pablo García Molina ${ }^{a}$.

aDepartamento de Enfermería, Facultad de Enfermería y Podología, Universidad de Valencia (Valencia, España), ${ }^{b}$ Hospital Clínico Universitario de Valencia (Valencia, España), 'Servicio de Emergencias Sanitarias SES-SAMU (Valencia, España) y ${ }^{\mathrm{d} E s c u e l a ~ d e ~ E n f e r m e r i ́ a ~ d e ~ L a ~ F e, ~ U n i v e r s i d a d ~ d e ~ V a l e n c i a ~(V a l e n c i a, ~ E s p a n ̃ a) . ~}$

\section{\$EWWDWW}

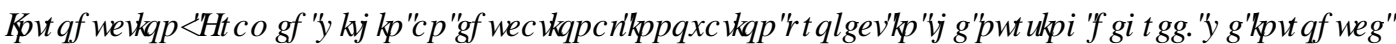

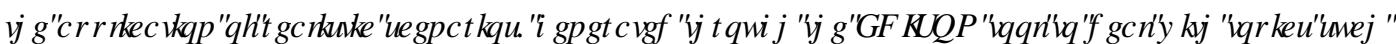

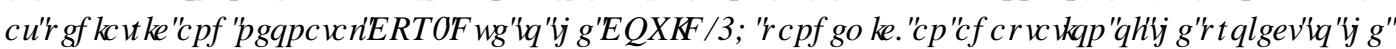
WHPP DUFIP RGDOWZ DVSLRSRUHGD

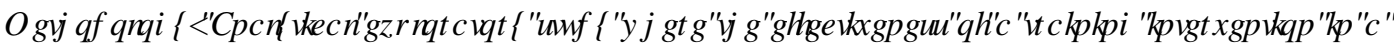

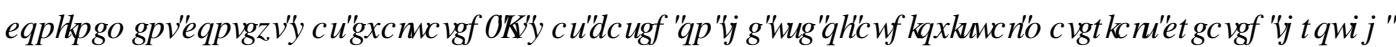

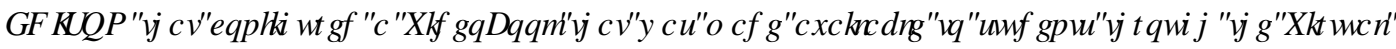

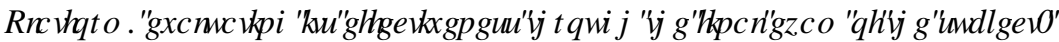

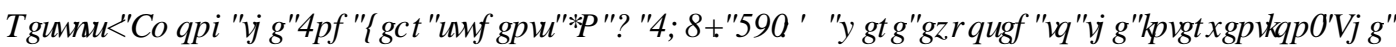

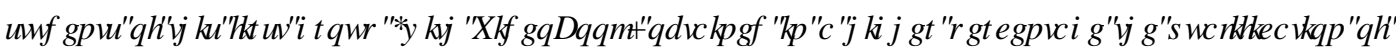
3 ( [FHOQWH

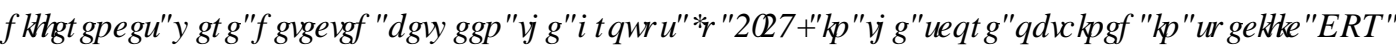

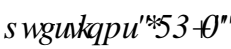

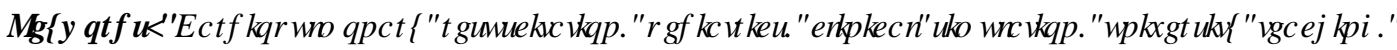

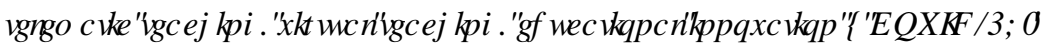

\section{HXP HQ}

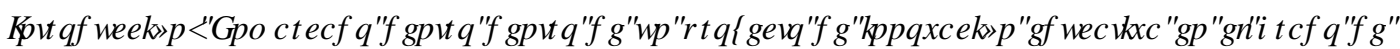

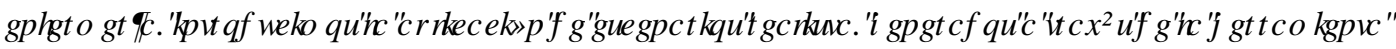

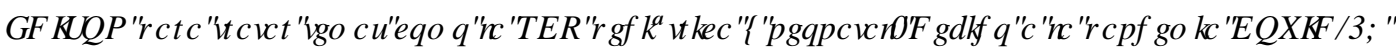

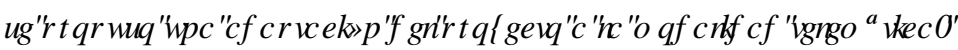

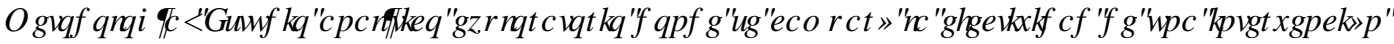

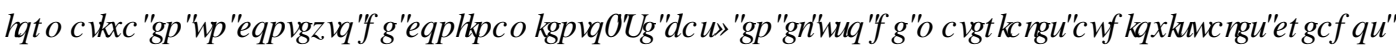

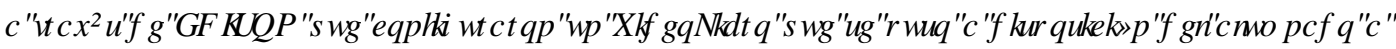

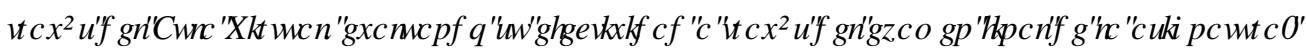

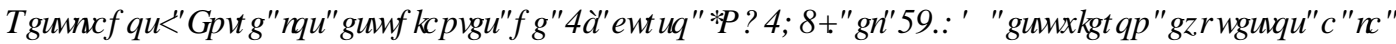

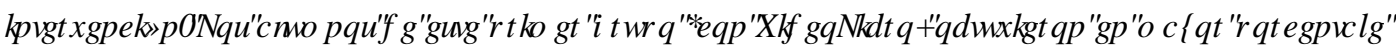

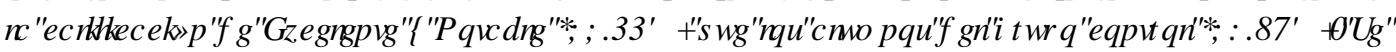

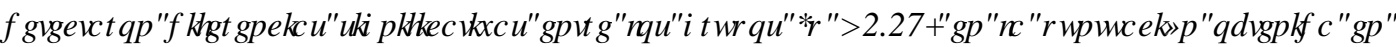

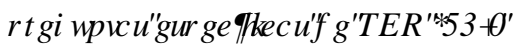

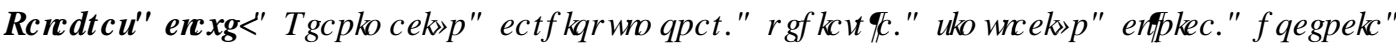

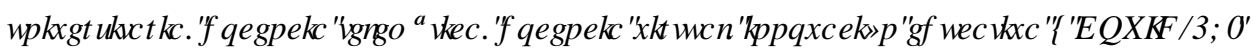




\section{Introducción}

El programa de Innovación Educativa en Reanimación Cardiopulmonar básica y avanzada en pediatría y neonatología en un contexto realista, se incluye dentro de la asignatura "Enfermería en la Salud Infantil y Adolescente" (ESIA), impartida en $2^{\circ}$ curso del grado en Enfermería de la Facultad de Enfermería y Podología (FIP) de la Universidad de Valencia. Se enmarca en un proyecto de Innovación Educativa y Mejora de la calidad docente, del Servicio de Formación Permanente e Innovación Educativa (SFPIE) de la Universidad deValencia (Uv.es, 2017).

A lo largo del recorrido de este proyecto, en los anteriores cursos académicos, se diseñaron diferentes situaciones clínicas del contexto crítico pediátrico y neonatal, en las que los alumnos y alumnas de la asignatura ESIA tenían que enfrentarse a diversos escenarios donde poner en práctica de manera dinámica los conocimientos, habilidades y actitudes adquiridos a lo largo del curso académico. Este tipo de formación se fundamenta en que el nuevo paradigma educativo, que requiere una formación del alumnado basada en la competencia y para ello hay que incorporar nuevos materiales, nuevas metodologías e introducir prácticas en la docencia, consiguiendo de esta manera mejoras en el proceso de enseñanza-aprendizaje (Calveras, 2003).

El profesorado de enfermería no puede pretender que el alumnado aprenda a resolver situaciones de su futura práctica diaria, solamente a través de la observación del profesor o documentos "pasivos" basados en presentaciones. El alumnado debe ser expuesto a situaciones clínicas donde puedan discutir acerca de las posibles soluciones, para posteriormente tomar decisiones. Por ello, es importante la incorporación de la simulación clínica junto con herramientas de entorno 3D en la docencia de enfermería (Guimond, Sole, \& Salas, 2011; Urra, Sandoval, \& Irribarren, 2017; Jeffries, 2005).

Este tipo de metodología docente es importante para la formación en el manejo adecuado de una situación de emergencia, ya que ésta es muy difícil de entrenar y evaluar durante la emergencia real. Cuando se produce una situación crítica no hay tiempo para pararse y pensar sobre cual será el próximo paso, por este motivo es imprescindible practicar el protocolo de actuación, para saber qué acciones hay que llevar a cabo.

Por esta razón, la programación docente de la asignatura dedica a la Reanimación Cardiopulmonar una sesión teórica previa, con una duración de dos horas y un grupo de 80 alumnos, y un laboratorio práctico de otras dos horas en grupos reducidos con 15 alumnos. Esto permite que pongan en práctica los conocimientos impartidos y los consoliden, como se pudo demostrar en anteriores fases del proyecto de innovación (Tortajada-Lohaces, 2018; García-Molina, 2018 y 2019). Además, la simulación constituye una excelente forma de practicar una situación de emergencia (en este caso la reanimación cardiopulmonar) sin poner en peligro la vida del paciente mediante un ambiente realista generado en 3D (De la Horra, 2010).

La creación de estos escenarios realista, se genera a través de la herramienta EDISON, que permite la grabación de video y posterior edición, la trasmisión remota en streming y la modalidad presencial en vivo. Para la aplicación de este software, tan solo es necesario contar con un ordenador portátil, un dispositivo de captura de video, un fondo de chroma y un dispositivo de interacción remota. Una vez comenzada la trasmisión de video, el programa incluye la imagen del presentador en el fondo predefinido, tales como una presentación de diapositivas o una grabación de una simulación clínica.

En definitiva, la docencia virtual o telemática, se ve enriquecida con la aplicación de estos entornos en 3D que permite dotar de un nivel de vivencia y percepción de la gravedad del paciente pediátrico y neonatal ante una actuación futura de este calibre. También, el hecho de que los alumnos de cursos más avanzados utilicen la herramienta EDISON para tratar temas como la RCP pediátrica y neonatal (coordinados por profesionales sanitarios especialistas) permitirá dotarles de las competencias esenciales para poder ser ellas mismas quienes formen a los alumnos/as de $2^{\circ}$ curso. 


\section{Objetivos}

- Formar al alumnado en Reanimación Cardiopulmonar Pediátrica y Neonatal de forma realista a través de la docencia virtual.

- Evaluar los conocimientos sobre RCP pediátrica después de la intervención educativa (EDISON online).

- Involucrar al alumnado de tercer y cuarto en la docencia telemática impartida con la tecnología EDISON.

\section{Desarrollo de la innovación}

Este Proyecto de Innovación Docente (PID) fue aceptado por el Vicerectorat d'Ocupació i Programes Formatius de la Universitat de Valencia en el año 2019 para su realización en dos cursos 2019-2021, con el código de proyecto UV-SFPIE_PID19-1096189 y con una financiación de 1000 euros.

El curso 2019-2020, estuvo marcado por la aparición y llegada a nuestro país del $6 \$ 56 \square \& R 9 \square$ que tuvo como consecuencia el confinamiento del alumnado y el profesorado. El equipo de innovación rediseñó entonces la metodología, adaptándola a esta nueva situación. Se facilitaron vídeos demostrativos simulados sobre las técnicas de RCP, grabados y editados previamente por el equipo docente de la asignatura. Además se ofertaron tanto documentación de apoyo como las videoconferencias de forma síncrona y se permitió la grabación de la clase a todo el alumnado de $2^{\circ}$ curso. Estas clases fueron ofrecidas por el alumnado de $4^{\circ}$ curso, tutorizado por un profesor de la asignatura. Estos recursos didácticos se ofrecieron a todo el alumnado.

Como se pretendía evaluar el valor de la aplicación de entornos virtuales atractivos creados con EDISON en la educación de RCP, se crearon materiales audiovisuales organizados como un "videolibro". Opción que se puede encontrar en el AulaVirtual (basada en el entorno MODDLE). Como introdujimos antes, "Edison" es una herramienta interactiva para la creación de presentaciones que incrementa la atención de los/as estudiantes y ayuda a la transmisión del mensaje mediante la inmersión del docente dentro de su presentación en un entorno 3D, véase como ejemplo la ) $L X W \square \square$

\section{Compresiones torácicas en $\geq 1$ año}

- Colocar el talón un través de dedo encima del apófisis xifoides.

- Colocarse en la vertical del pecho.

- Mantener el brazo extendido y comprimir el esternón.

- Dedos de la mano elevados.

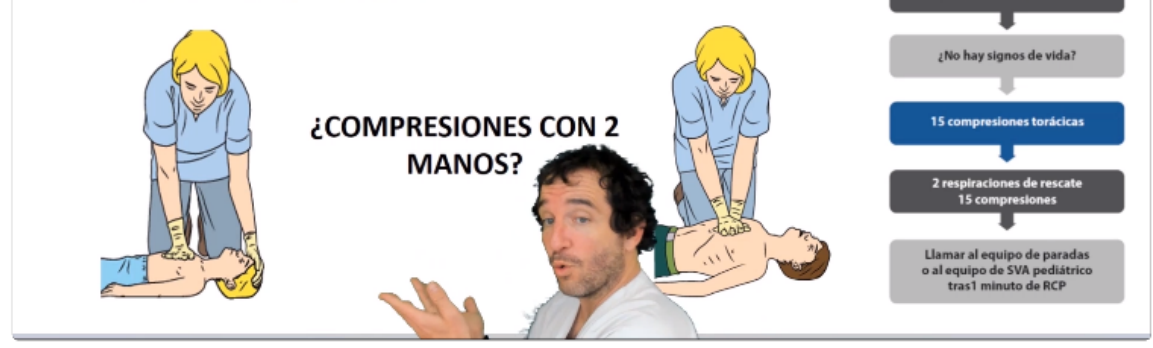

) 
La aplicación está basada en plantillas, que permite a los docentes transformar el proceso de aprendizaje buscando crear una experiencia educativa diferente, que permita tanto a estudiantes como docentes interactuar con gráficos de realidad aumentada inmersos en entornos virtuales personalizables y completar las lecciones con elementos 3D predefinidos. El profesorado pudo explicar conceptos complicados de una manera sencilla, visual y práctica. Una vez cargada la plantilla, el programa inserta al docente, capturado por la cámara del portátil, dentro de la escena de realidad aumentada, que se puede personalizar usando los diferentes recursos y objetos disponibles, véanse ) LXXWU⿴囗十丁
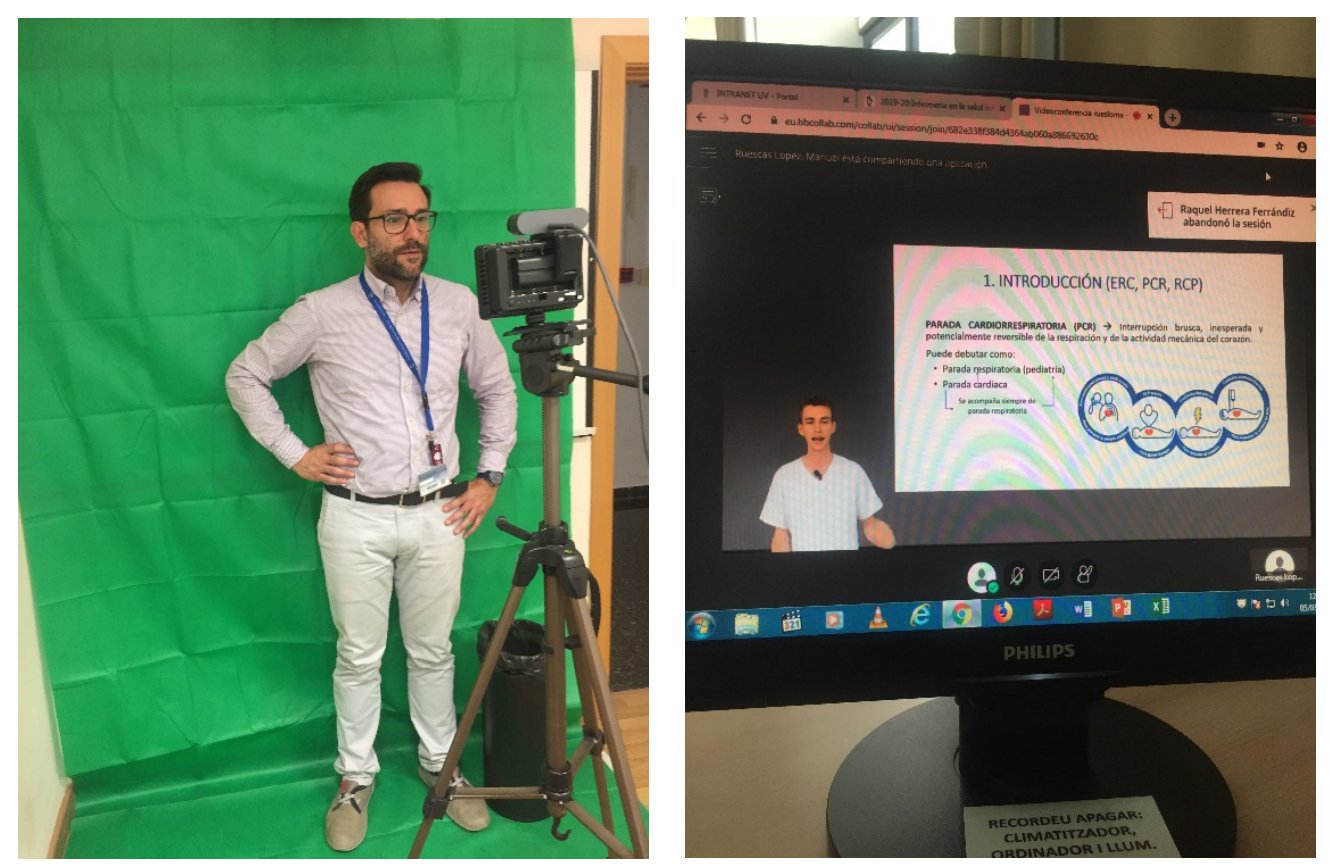

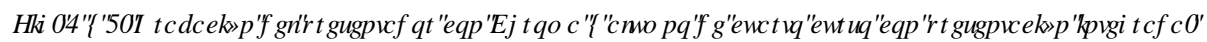

Como el videolibro se hizo en castellano, se decidió utilizar en los grupos donde el castellano era la lengua utilizada en las clases y materiales docentes प) IJXWDQ. 2 grupos (castellano) tuvieron docencia en el AulaVirtual con videolibro (CVL), mientras que otros 3 grupos (valenciano) estuvieron sin videolibro (SVL), pero con el resto de recursos que también tenían los otros 2 grupos. La efectividad del videolibro se midió a través de las preguntas sobre RCP que se formularon en el examen final de la asignatura en junio de 2020. Se formularon las mismas 10 preguntas a todo el alumnado dentro de un examen de 55 preguntas. Se valoró si hubieron diferencias significativas entre los grupos, tanto en las 10 preguntas sobre RCP, como en el conjunto del examen.

\section{RCP básica y avanzada, OVACE y organización en PCR pediàtrica y neonatall}

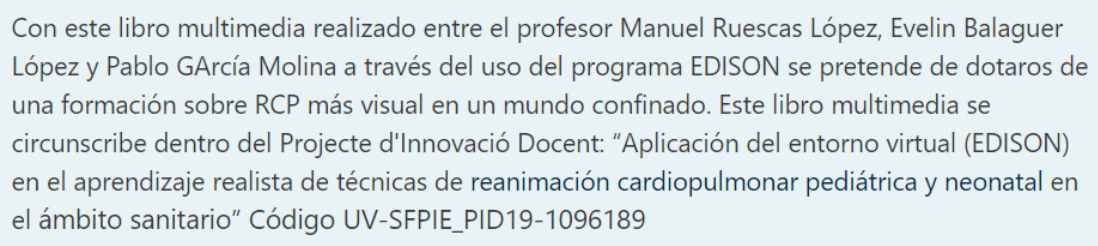

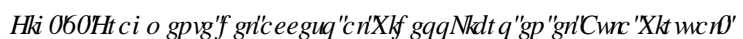




\section{Resultados}

El proyecto contó con la participaron 296 estudiantes. El 37,8\% (112) de los alumnos recibieron formación CVL. La nota media final del examen fue para el grupo CVL de 8,44 (DE 0,55), mientras que para el grupo SVL fue de 8,62 (DE 0,54).

Existen diferencias significativas $(\mathrm{p}=0,005)$ en las notas numéricas obtenidas entre los alumnos CVL y los alumnos SVL, siendo mayor la media numérica de los alumnos SVL. Sin embargo, a la hora de trasladar esas puntuaciones numéricas a la calificación categórica, los alumnos CVL obtuvieron en mayor porcentaje la calificcación de Excelente y Notable (99,11\% de las calificaciones) que los alumnos SVL (98,65\%).

7DECD П5 HXQWDRVFDUJ Y UFRVGHOH DP HQHQDP ERVJUXSRV

\begin{tabular}{llll}
\hline Etiquetas de fila & CVL & SVL & Total general \\
\hline Bien & $1(0,89 \%)$ & $3(1,63 \%)$ & $4(1,35 \%)$ \\
Excelente & $101(90,18 \%)$ & $162(88,04 \%)$ & $263(88,85 \%)$ \\
Notable & $10(8,93 \%)$ & $19(10,33 \%)$ & $29(9,80 \%)$ \\
Total general & 112 & 184 & 296 \\
\hline
\end{tabular}

) XHQUALC (DERUFIYQSLRSID

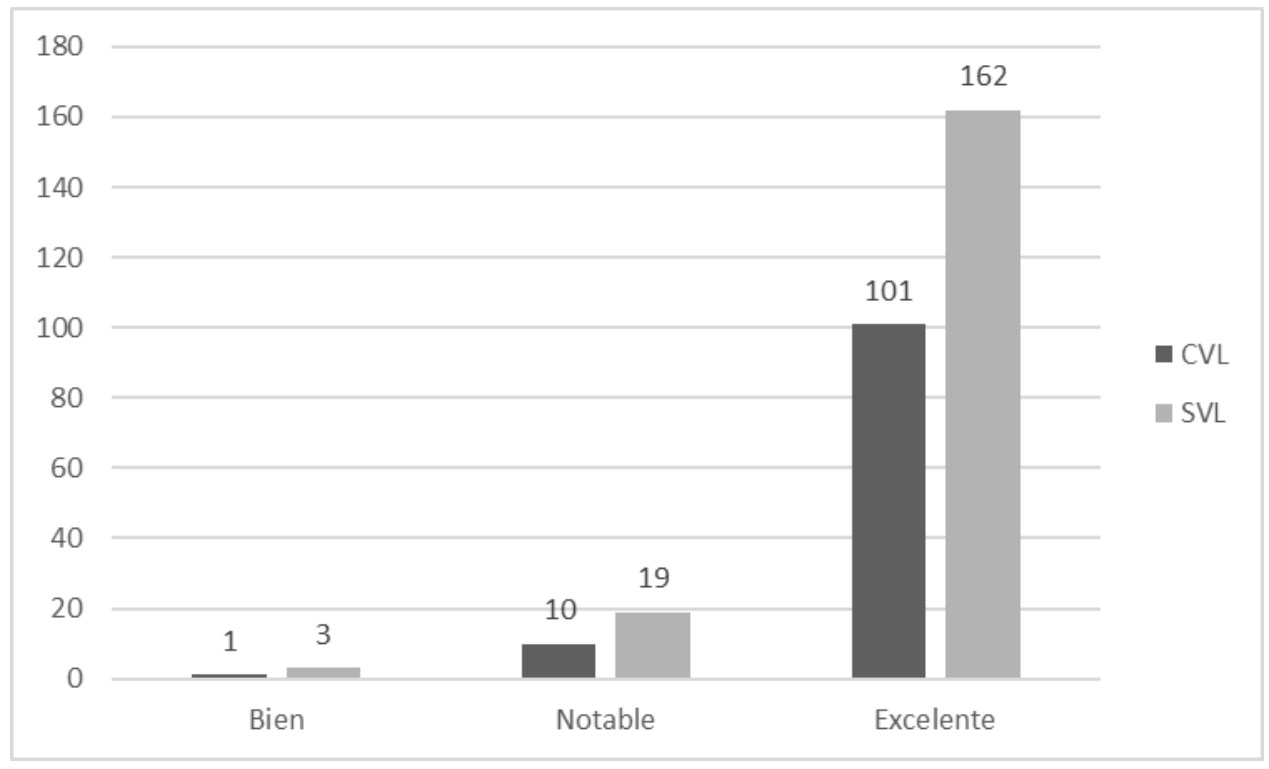

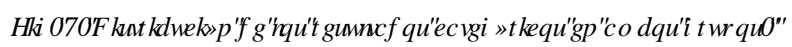


Al evaluar en qué preguntas se daban diferencias significativas en la puntuación obtenida se observa que la pregunta 31 obtiene una menor media de puntuación en el grupo CVL que en el grupo SVL.

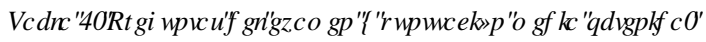

\begin{tabular}{lccc}
\hline Pregunta & CVL & SVL & P \\
\hline $\begin{array}{l}\text { ¿Cuál es la relación compresiones-ventilaciones que debes hacer a un } \\
\text { niño de 2 meses de edad? }\end{array}$ & 0,1982 & 0,1913 & 0,05 \\
$\begin{array}{l}\text { ¿Cada cuánto tiempo se pone la adrenalina en la rcp avanzada? } \\
\begin{array}{l}\text { ¿Cuál será la pauta de medicación a administrar en un niño de 3 años } \\
\text { que está convulsionando, antes de ser considerado en estatus } \\
\text { convulsivo? }\end{array}\end{array}$ & 0,1946 & 0,1804 & 0,08 \\
$\begin{array}{l}\text { A maría le tienes que administrar 185 mg de vancomicina en una } \\
\text { dilución segura de 5mg/ml. Reconstituyes el vial de 500 mg de } \\
\text { vancomicina en 10 ml de h2o para inyeccion ¿en cuánto volumen } \\
\text { debes administrar el fármaco para no provocar flebitis? }\end{array}$ & 0,1478 & 0,00 \\
\hline
\end{tabular}

\section{) XHQWILC QDERUFIYQSLRSIDI}

El resto de preguntas del cuestionario de evaluación no obtuvo diferencias significativas en la puntuación media obtenida entre ambos grupos.

Como discusión de los resultados, la incorporación de la simulación clínica junto con herramientas de entorno 3D en la docencia de enfermería, aumentan el grado en que el alumnado adquiere los objetivos de aprendizaje (Guimond, Sole, \& Salas, 2011; Urra, Sandoval, \& Irribarren, 2017; Jeffries, 2005). Este hecho lo hemos podido constatar con esta investigación, que ha demostrado como en nuestra población a estudio mejoraban las calificaciones categóricas en el grupo que dispuso del Videolibro, a diferencia del que no.

Se ha podido ver la utilidad de EDISON como herramienta para la metodología de Aula Invertida y en la creación de escenarios reales en un contexto de confinamiento domiciliario. Además, gracias a la inclusión de alumnado de tercer y cuarto curso en el proyecto hemos favorecido el aprendizaje entre iguales y les hemos ayudado a refrescar y consolidar los conocimientos que aprendieron cuando cursaron la asignatura.

Por otro lado, deben señalarse algunas limitaciones del estudio relacionadas con su metodología. En primer lugar no podemos establecer una relación directa entre la calificación obtenida en el examen final de la asignatura y la presencia del libro audiovisual, pues existen terceras variables que pueden actuar como factores de confusión, tales como las horas de dedicación al estudio por parte del alumnado, la visualización de otros recursos o la situación en la que realizaron la prueba final desde sus casas (acompañados, nerviosos, consulta de material, etc.).

Existió un sesgo en la selección del alumnado, ya que como el Videolibro era en español, se seleccionó los grupos en español. Mientras que los 3 grupos restantes eran en valenciano y no recibieron el Videolibro. Esta asignación por conveniencia es un sesgo a valorar y que se podría evitar creando Videolibros en valenciano y de esa forma poder aleatorizar la muestra.

Por último, existe un último riesgo relacionado con la visualización o no del VideoLibro en el grupo intervención (CVL), pues no podemos estar completamente seguro de si el alumnado a estudio visualizó o no el recurso antes de la prueba final. En futuros estudios sería necesario monitorizar la intervención para 
asegurarnos de que es visualizada por todos los alumnos del grupo o excluir del análisis a aquellos que no lo hicieron.

\section{Conclusiones}

Nuestro estudio tuvo un carácter exploratorio analítico y nos permitió reconocer el recurso didáctico de grabación EDISON como una innovadora herramienta para la docencia virtual, tanto para las sesiones teóricas síncronas como para la recreación de escenarios de simulación clínica.

Los resultados fueron satisfactorios, pero aún queda un largo camino por recorrer y no hay que darse por satisfechos con los logros obtenidos. Se debe continuar implementando la herramienta en la docencia de la asignatura para, así aumentar la gama de recursos audiovisuales, haciendo especial hincapié en la grabación de escenarios de reanimación cardiopulmonar realistas donde el alumnado se pueda ver sumergido.

\section{Referencias}

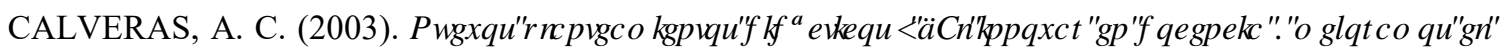
DSUHQ] DNH' , 18-21.

DE LA HORRA GUTIÉRREZ, I. (2010). "La simulación clínica como herramienta de evaluación de competencias en la formación enfermera". [En 5HGXFD Universidad Complutense de Madrid.

GARCÍA-MOLINA, P., BALAGUER-LÓPEZ, E., PATIÑO-SERRA, M., TORTAJADA-LOHACES, A., SANCHIS-SÁNCHEZ, E., SÁNCHEZ-LORENTE, M., \& BLASCO-IGUAL, J. (2019). "Clinical simulation: innovative educational project in basic cardiopulmonary resuscitation and advanced in

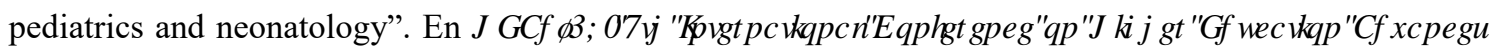
(pp. 1233-1240). Editorial Universitat Politècnica de València.

GARCÍA-MOLINA, P., BLASCO-IGUAL, J. M., BALAGUER-LÓPEZ, E., TORTAJADA-LOHACES, A., SANCHIS-SANCHEZ, E., GEORGIEVA, S., \& SÁNCHEZ-LORENTE, M. M. (2018ם“"Educational innovation in basic and advanced cardiopulmonary resuscitation in pediatrics and neonatology in a realistic

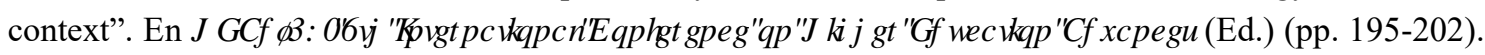
Editorial Universitat Politècnica de València. https://doi.org/http://dx.doi.org/10.4995/HEAd18.2018.7945

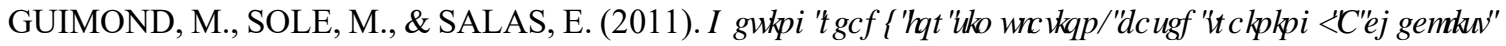
IRUQXUHHGXFDURWW Nurs Educ Perspect, 32(3), 179-185.

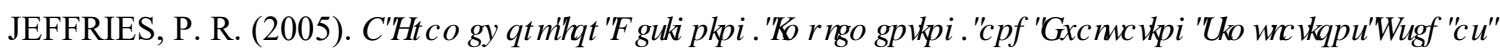

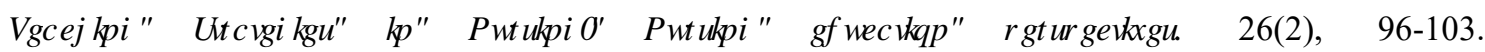
https://doi.org/10.1043/1536-5026(2005)026<0096:AFWFDI>2.0.CO;2

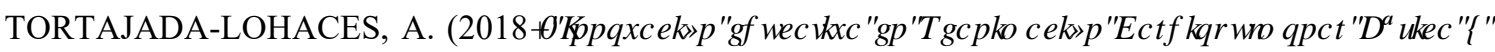

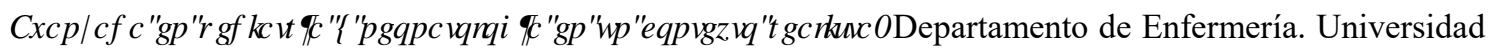
de Valencia.

URRA MEDINA, E., SANDOVAL BARRIENTOS, S., \& IRRIBARREN NAVARRO, F. (2017). ( 0

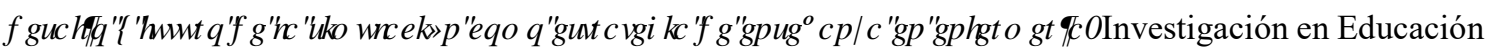
Médica, 6(22), 119-125. https://doi.org/10.1016/j.riem.2017.01.147

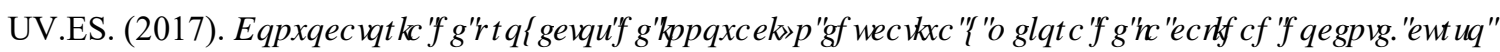

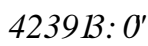

